\title{
Trapping of discrete solitons by defects in nonlinear waveguide arrays
}

\author{
Luis Morales-Molina and Rodrigo A. Vicencio \\ Max-Planck-Institut für Physik komplexer Systeme, \\ Nöthnitzer Strasse 38, D-01187 Dresden, Germany
}

\begin{abstract}
We study the trapping process of moving discrete solitons by linear and nonlinear impurities embedded in a one-dimensional nonlinear cubic array. We show that there exist optimal values for the strength of impurity and the angle for which a strong trapping is obtained. We introduce a criterion for studying scattering dynamics of localized waves in nonlinear extended systems, where trapping of energy takes place.
\end{abstract}

(c) 2005 Optical Society of America

OCIS codes: $190.0190,190.4370,190.5530$. 
The study of nonlinear waves and in particular the trapping of moving localized nonlinear waves is a phenomenon that is receiving a lot of attention. Most of the interest is because of the potential applications in different branches of physics ranging from nonlinear optics to Bose-Einstein condensates ${ }^{1}$. The interplay between discreteness and nonlinearity gives rise to the formation of localized structures, the so-called discrete solitons. These entities were theroretically predicted ${ }^{2}$ and experimentally observed ${ }^{3}$ in nonlinear optical waveguide arrays. Its implementation has been suggested to be useful in the development of multiport switching systems for future all-optical technologies ${ }^{4}$.

The scattering of discrete solitons by impurities has been discussed by Królikowski and Kivshar in Ref.5. They showed that it is possible to trap a moving soliton by using a local change in the coupling between two neighbor waveguides. On the other hand, in the context of gap solitons it has been recently shown (in the scattering process of solitons by defects) that trapping of light takes place because of the resonant transfer of energy from the soliton to the defect mode ${ }^{6}$. This is a very remarkable effect which could represent a fundamental stage in the process of steering of solitons in all optical devices ${ }^{7}$. In this respect, it would be very desirable, from the application point of view to make optimal the trapping mechanism.

In the present letter we characterize the scattering process of solitons by linear (LI) and nonlinear (NLI) impurities located at one site of the array. In order to provide a full description of the scattering process we introduce a new criterion which takes into account the trapping of moving localized waves. The main difference with the standard definition resides on the fact that it allows to measure besides those energies reflected and transmitted, that energy trapped after the scattering process with defects. As a main result of our description is that it provides the basic ideas for tuning the optimal parameters in order to get a maximum trapping. A second main result derived from our analysis reveals that, the 
trapping of solitons is indeed a consequence of a resonant transfer of energy between the impurity mode and the soliton as suggested in Ref.6.

The evolution of guided-mode fields in a one-dimensional nonlinear cubic waveguide array $^{2}$ in the presence of defects, can be described by the perturbed discrete nonlinear Schrödinger equation

$$
i \frac{d u_{n}}{d \xi}+\left(u_{n+1}+u_{n-1}\right)+\epsilon_{1} u_{n} \delta_{n, i_{0}}+\left(1+\epsilon_{2} \delta_{n, i_{0}}\right)\left|u_{n}\right|^{2} u_{n}=0,
$$

where $u_{n}$ is the field amplitude in the $n$th waveguide. $\xi$ is the dimensionless propagation distance; $\epsilon_{1}$ and $\epsilon_{2}$ represent the strength of linear and nonlinear defects, respectively. $\delta_{n, i_{0}}$ is the delta's Kronecker, and $i_{0}$ denotes the site where the impurity is located. The realization of this model can be done in different experimental setups. In waveguide arrays, the simple way to implement defects is changing locally the refraction index by doping a particular waveguide with different materials or by changing the geometry ${ }^{3}$. Another possible setup can be realized in arrays of nondiffracting Bessel beams in Kerr-type nonlinear media ${ }^{8}$. In this case one can modify the linear and nonlinear properties by changing locally the depth of refractive index modulation and the Bessel beam's parameters, respectively.

For the numerical investigation of the trapping process we use as an initial condition the discrete version of the NLSE's exact solution ${ }^{4,5}$ given by: $u_{n}(0)=$ $A$ sech $[A(n-n c) / \sqrt{2}] e^{i k(n-n c)}$. Here $A$ is the soliton amplitude which is also related to the inverse of the soliton width; $k$ is the initial kick (transversal angle) and $n c$ is the site where the initial condition takes the maximum value in an array formed by $N=201$ sites. Generally speaking, all the numerical experiments realized throughout the paper consist in launching a soliton with the previous ansatz against the impurity site. Since we want to study the trapping of moving solitons by defects we should be able to find a balance between discreteness of the lattice and mobility of the solitons. Lattice discreteness favors the 
trapping of solitons but, on the other hand, for a high amplitude, the mobility of the soliton is practically reduced to zero because of the so-called Peierls-Nabarro potential ${ }^{9}$. Hence, the ideal configuration for the analysis of trapping is found in an intermediate regime where the soliton still presents high mobility. Consequently, we set $A=0.7$ for all our numerical computations.

Now, once the soliton collides with the defect we may have either pure transmission, trapping, reflection or simply a mixed state of the previous ones. The main issue here is how to quantify the trapped energy (power) and at the same time to distinguish this power from those reflected and transmitted in different cases. In this respect, aimed to fulfill the above requirement, we introduce the following more general definition

$$
R+T+L=1
$$

for the description, in principle, of any nonlinear scattering problem. Here $R$, $T$, and $L$, represent the percentages of the initial power that it is reflected, transmitted, and localized (trapped), respectively. We define trapping as: $L=\sum_{n=i_{0}-10}^{i_{0}+10}\left|u_{n}\right|^{2} / \sum_{n=1}^{N}\left|u_{n}\right|^{2} . R$ is measured from $n=1$ up to $n=i_{0}-11$, and $T$ from $n=i_{0}+11$ up to $N$. In case of no defect $(L=0)$ the previous equation reduces to the standard expression $R+T=1$, commonly used to quantify the scattering process of waves. According to this definition, it is considered as trapped, the amount of power bounded in the interval $\left\{i_{0}-10, i_{0}+10\right\}$ around the defect. The size of this interval depends on how narrow is the region where the soliton remains trapped and it is equivalent to the width of the impurity mode ${ }^{10}$.

Now, we proceed to compute the value of $L$ doing sweeps in the $\{k, \epsilon\}$ space of parameters. In what follows, we restrict our analysis to individual (LI or NLI) impurity cases. From Fig.1, we see a very different behavior for linear and nonlinear cases. In the linear case, one observe a smooth decay of trapping as we take larger values for $\epsilon$. Accordingly, the 
borders of the trapping region in the parameter space become fuzzy, thus being impossible to distinguish between the power trapped and those transmitted and reflected. Interestingly, for the linear case highlights the absence of trapping for strong strength of the defect and small $k$. Intuitively, one would expect more trapping as the strength of the defect increases. In contrast to the linear case, for the nonlinear case it is noticeable the existence of a region with well defined borders in the parameter space where takes place a strong trapping. However, the most striking feature is the bifurcation of the trapping region in two branches. The narrow branch splits sharply the parameter space in transmission, trapping and reflection regions. This means that by fixing either $k$ or $\epsilon$ and varying the other parameter one may observe more than one window of trapping. This result reinforces previous suggestion that trapping is a result of a resonant interaction phenomenom between the impurity mode and the soliton ${ }^{6}$. Regarding the formation of bifurcations, we have observed for a higher order of the nonlinearity of the impurity the appearance of more than one bifurcation of the trapping region, which indicates the importance of the nature of the impurity mode in the trapping process.

In order to do a better description of Fig.1 a) and b) we simplify the analysis by fixing a value of the angle for both linear and nonlinear cases. In Fig.1c are depicted the results of an $\epsilon$-sweep for $k=0.2$. This figure essentially shows two differences between linear and nonlinear impurities. First, the maximum trapping is approximately $96 \%$ of the incoming power for the nonlinear case while in the linear case the trapping reaches a $84 \%$. A second main difference is concerning the phenomenology in the scattering process. In the linear case, coming from zero value of $\epsilon$ up to values close to the first maximum, essentially transmission takes place. However, as we get closer to the first maximum a mixed state between transmission and trapping appears with an increasing trapping. So, at the maximum, most 
of the power is trapped at the impurity site (see Fig.2a). Afterwards the behavior starts to change, by observing a mixture between a decaying trapping and an increasing reflection [Fig.2d]. Similar reflection has been recently pointed out in the context of Bose-Einstein condensates $^{11}$. The complete description of the scattering process in the linear defect case is summarized as follows: $T \rightarrow T+L \rightarrow L+R \rightarrow R$. In the nonlinear impurity case the phenomenology is rather different. Approximately below $\epsilon=0.75$ it is possible to observe a succession of pure transmission, trapping (see e.g. Fig.2b) and reflection effects as long as increases the defect strength. It is worth to mention that after the first maximum we have a nearly perfect reflection similar to that depicted in Fig.2d. However, above the value $\epsilon \approx 0.75$ we observe a complete different behavior. Instead of a total reflection, we observe a sort of "bouncing" effect [Fig.2c]. Similar behavior has also been observed for gap solitons ${ }^{6}$ Briefly speaking, when the soliton arrives to the scattering region it starts to populate the impurity mode as expected. However, in this case due to the interaction, although the soliton is at first reflected it keeps barely moving in the vicinity of the defect for a relative large time instead of moving away, before it is finally trapped by the defect.

In both linear and nonlinear cases, we observe that once the soliton gets trapped into the impurity region, it begins to oscillate periodically with the profile of the impurity mode. These oscillations are visualized in Fig.2 as a periodic sequence of intermittent bright dots. Note that the distance between the bright dots is reduced in Fig.2c with respect to that depicted in Fig.2b. This indicates that the frequency of oscillations is directly related with the strength of the defect. From the above results one realize the importance of the role of the impurity mode in the scattering process. In this sense, some explanations in terms of the collective coordinate approach have been proposed for the scattering of solitons by a point-like impurity defect (see e.g. Ref. 12). This approach uses as additional degree of 
freedom the excitation of the impurity mode, which sheds light on some of the main features that we have previously shown. In few words, this approach describes the motion of the soliton center during its interaction with the defects. Essentially, the description reduces to a particle moving in an effective potential formed by two parts, one is a classical well and a second one which accounts for the soliton-nonlinear defect mode interaction. This latter term is a phase dependent function, whose phase in turn changes periodically in time, with a variation rate mainly determined by the intrinsic frequency of the impurity mode. In case that the soliton and the impurity mode are out of phase, the resulting potential acts as a barrier and the particle for relatively small velocities may turn back after reaching the turning point, namely the soliton is reflected. Otherwise, the particle can be either trapped if we consider that due to some phenomenological breathing motion effect ${ }^{13}$ the kinetic energy is reduced leading eventually the particle to remain into the well or transmitted for a particle with sufficient kinetic energy.

Finally, for a combination of both impurities no improvement of trapping has been observed compared to the single nonlinear case. The system behaves essentially as in the presence of a linear defect.

In summary, we have shown a fairly good method to estimate optimal experimental parameter values for which a strong trapping of moving localized waves takes place. In particular, for the nonlinear case, a bifurcation in the parameter space was observed, which adds new evidence over the existence of a resonance process between the soliton and the impurity mode in the trapping process. In this respect, we hope that these results encourage the realization of experiments addressed to check the validity of these theoretical predictions which could be of remarkable importance for technological purposes.

We thank G. Kalosakas and Y. Kivshar for useful comments. L.M.-M.'s e-mail address 
is lmolina@mpipks-dresden.mpg.de.

\section{References}

1. D.K. Cambpell, S. Flach, and Y.S. Kivshar, Physics Today 57, 43 (2004).

2. D. N. Christodoulides and R. I. Joseph, Opt. Lett. 13, 794 (1988).

3. H. S. Eisenberg et al., Phys. Rev. Lett. 81, 3383 (1998).

4. R. A. Vicencio, M. I. Molina, and Y. S. Kivshar, Opt. Lett. 29, 2905 (2004).

5. W. Królikowski and Y. S. Kivshar, J. Opt. Soc. Am. B 13, 8761996.

6. R.H. Goodman, R.E. Slusher and M.I. Weinstein, J. Opt. Soc. Am. B 191635 (2002).

7. R. Morandoti et al., Opt. Lett. 28, 834 (2003).

8. Zhiyong Xu, Y. V. Kartashov, and L. Torner, Opt. Express. 13, 1774 (2005).

9. Yu. S. Kivshar and D. K. Campbell, Phys. Rev. E 48, 3077 (1993).

10. G.Kalosakas et al., Phys. Rev. E 56, 1088 (1997).

11. C. Lee and J. Brand, cond-mat/0505697.

12. R.H. Goodman, P.J. Holmes and M.I. Weinstein, Physica D 192215 (2004).

13. H. Sakaguchi and M. Tamura, J. Phys. Soc. Japan 73, 503 (2004). 


\section{List of Figure Captions}

Fig. 1. a) and b) Trapping $L$ vs $k$ and $\epsilon$ : a) linear; b) nonlinear. c) Trapping $L$ vs $\epsilon$ for the value $k=0.2$ depicted by a dashed white line in Fig.1 a) and b). The results are depicted by diamonds and stars for the linear and nonlinear cases, respectively.

Fig. 2. Intensity evolution for different points in Fig.1c labeled by letters. a) LI, $\epsilon=0.3$. b) NLI, $\epsilon=0.42$. c) NLI, $\epsilon=0.92$. d) LI, $\epsilon=1$. 

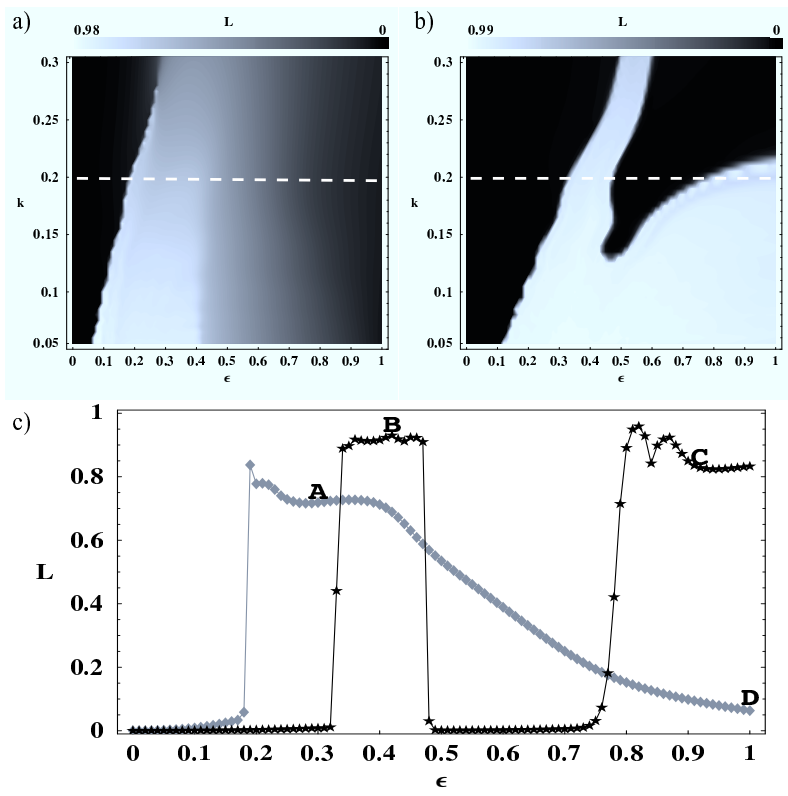

Fig. 1. a) and b) Trapping $L$ vs $k$ and $\epsilon$ : a) linear; b) nonlinear. c) Trapping $L$ vs $\epsilon$ for the value $k=0.2$ depicted by a dashed white line in Fig.1 a) and b). The results are depicted by diamonds and stars for the linear and nonlinear cases, respectively. 

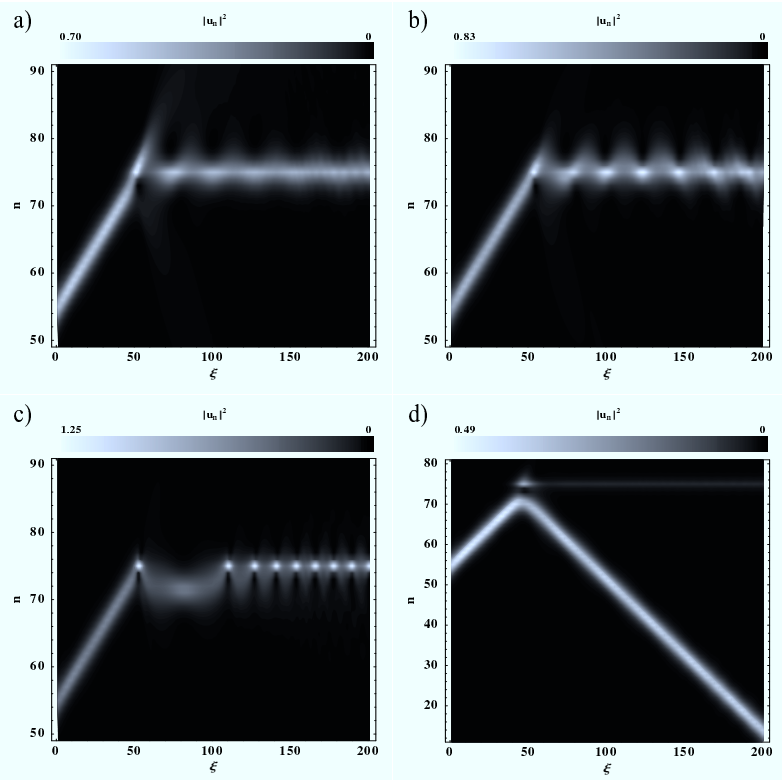

Fig. 2. Intensity evolution for different points in Fig.1c labeled by letters. a) LI, $\epsilon=0.3$. b) NLI, $\epsilon=0.42$. c) NLI, $\epsilon=0.92$. d) LI, $\epsilon=1$. 